\title{
Understanding the interactions between Eimeria infection and gut microbiota, towards the control of chicken coccidiosis: a review
}

\author{
Thabile Madlala, Moses Okpeku, and Matthew Adekunle Adeleke* \\ Discipline of Genetics, School of Life Sciences, College of Agriculture, Engineering and Science, University of KwaZulu-Natal, \\ Westville, P/Bag X54001, Durban 4000, South Africa
}

Received 29 August 2020, Accepted 14 May 2021, Published online 2 June 2021

\begin{abstract}
The gastrointestinal tract in poultry harbours a diverse microbial community that serves a crucial role in digestion and protection. Disruption of the gut environment due to Eimeria spp. parasite infection causes an imbalance in intestinal homeostasis, driving the increment of pathogens such as Clostridium species. Coccidiosis infection affects the composition and integrity of gut microbiota, resulting in elevated susceptibility to diseases that pose a serious threat to the overall health and productivity of chickens. Anticoccidial drugs have proven effective in curbing coccidiosis but with concerning drawbacks like drug resistance and drug residues in meat. The exploration of natural alternative strategies such as probiotics and phytochemicals is significant in controlling coccidiosis through modification and restoration of gut microbiota, without inducing drug resistance. Understanding the interaction between Eimeria parasites and gut microbiota is crucial for the control and prevention of coccidiosis, and the development of novel alternative treatments.
\end{abstract}

Key words: Coccidiosis, Eimeria, Chickens, Gut microbiota, Probiotics, Phytochemicals.

\begin{abstract}
Résumé - Comprendre l'interaction entre l'infection à Eimeria et le microbiote intestinal pour lutter contre la coccidiose du poulet : une synthèse. Le tractus gastro-intestinal de la volaille abrite une communauté microbienne diversifiée qui joue un rôle crucial dans la digestion et la protection. La perturbation de l'environnement intestinal due à l'infection par le parasite Eimeria spp. provoque un déséquilibre de l'homéostasie intestinale, entraînant une prolifération des agents pathogènes tels que les espèces de Clostridium. L'infection par la coccidiose affecte la composition et l'intégrité du microbiote intestinal, ce qui entraîne une sensibilité élevée aux maladies qui ont un impact sérieux sur la santé globale et la productivité des poulets. Les médicaments anticoccidiens se sont avérés efficaces pour freiner la coccidiose, mais avec des effets négatifs inquiétants comme la résistance aux médicaments et la présence de résidus de médicaments dans la viande. L'exploration de stratégies alternatives naturelles telles que les probiotiques et les composés phytochimiques est importante pour le contrôle de la coccidiose par la modification et la restauration du microbiote intestinal, sans induire de résistance aux médicaments. Comprendre l'interaction entre les parasites Eimeria et le microbiote intestinal est crucial pour le contrôle et la prévention de la coccidiose et le développement de nouveaux traitements alternatifs.
\end{abstract}

\section{Introduction}

Coccidiosis is the most significant ubiquitous disease in the chicken industry globally caused by obligate intracellular parasitic protozoa belonging to the genus Eimeria. It currently results in great economic losses exceeding USD 3 billion yearly due to loss of productivity, high mortality and high treatment costs to control the disease [76, 93]. The disease is characterised by the colonisation and overcrowding of intestinal mucosa of chickens by Eimeria spp., compromising chicken wellbeing and productivity. Infections caused by Eimeria can easily be

\footnotetext{
*Corresponding Author: adelekem@ukzn. ac.za
}

transmitted between hosts by the direct faecal-oral route through ingestion of sporulated oocysts from contaminated feed or litter [27, 45]. Seven recognised Eimeria species cause coccidiosis at varying degrees of severity in chickens [63]. These species include E. acervulina, E. brunetti, E. maxima, E. mitis, E. necatrix, E. praecox, and the most prevalent E. tenella [75]. Each species has its own host-specific spectrum, targeting specific sites in the gut and posing severe damage to the host's absorption capability and growth [37]. Turk [80] reported on colonisation sites preferred by Eimeria spp., with the upper small intestine (jejunum and duodenum) mainly infected by E. acervulina, E. necatrix, E. maxima and E. praecox. Eimeria tenella, E. mitis, and E. brunetti infections 
occur in the caecum, ileum and colon, where the reaction of intestinal lymphoid tissue and intestinal damage by E. tenella results in significant blood loss at the acute stages of infection, observed as haemorrhagic patches, an increase in caecal protein and DNA which affects chicken weight gain and reduction in muscle mass [21, 44].

Eimeria infection destroys host mucosal cells resulting in elevated cell permeability, nutrient and plasma protein leakage, impaired digestion, and protein absorption, contributing to clinical and subclinical effects of coccidiosis [54, 81, 90]. This compromises chicken wellbeing as it disrupts host gut homeostasis, causing significant malabsorption, reduced feed conversion and weight gain, and overall decreased productivity in chickens [44, 63]. The severe intestinal damage caused by Eimeria colonisation not only affects epithelial cells, but it causes great disruption of gut microbial communities in the gastrointestinal tract (GIT), promoting colonisation and proliferation of other pathogens such as Clostridium perfringens, causing susceptibility of infected chickens to secondary diseases, thus increasing chicken mortality [3, 27, 49]. Eimeria invasion results in an imbalance in the gut microbial community known as dysbiosis [18]. The GIT consists of diverse and significant microbes that aid in the nutrition and proper development of chickens. The control of these diseases involves high costs of treatment strategies. This has led to the exploration of various coccidial treatment strategies over the years, such as implementing chemoprophylaxis and live-attenuated vaccines. However, these strategies have proven to be effective with significant drawbacks, including drug resistance and possible parasite reversion to its virulent state. This review aims to understand the interaction between Eimeria infection and gut microbiota. Alternative strategies that are safe, antibiotic-free, and costeffective to control coccidiosis in poultry are also reviewed.

\section{The function of gastrointestinal gut microbiota}

The GIT of the chicken serves a critical role in digestion and protection. It is crucial in converting ingested feeds into nutrients essential for maintenance, growth, and reproduction [6]. It also aids in developing the immune response needed to prevent intestinal colonisation by pathogenic or opportunistic microorganisms through pathogen exclusion [30, 76, 79, 87]. The GIT comprises a complex and diverse microbiota in chickens, including bacteria, viruses, archaea, and fungi. Bacteria are predominant in the GIT and have a beneficial symbiotic interaction with the host, crucial for chicken nutrition, health and product ion [70]. These microorganisms attach to the epithelial wall and trigger the immune system's maturation consisting of the mucus layer, epithelial monolayer, and other immune cells. The layers form a protective barrier to combat colonisation by opportunistic bacteria [6]. They also produce vitamins (vitamin $\mathrm{K}$ ), short-chain fatty acids (acetic acid), organic acids (lactic acid) and other complex compounds that provide nutrients and energy essential for the nutrition and protection of the animal [76].

Microbes located in the GIT mainly maintain homeostasis of the intestinal mucosa by digestion of food sources, providing the energy needed to induce the intestinal immune system to fight against aggressions of other microorganisms [14, 86]. A standard or balanced gut microbiota reduces host susceptibility to pathogenic parasites like Eimeria spp. [30]. Commensal bacteria regulate nutrient absorption and protect the host by preventing pathogen colonisation through competitive exclusion [89]. They also regulate immune activity by controlling mediators secreted by the mucosa membrane, triggering helper cells $[61,79]$. A fully developed immune system secretes proteins such as immunoglobin $\mathrm{A}(\operatorname{Ig} \mathrm{A})$, essential for regulating bacterial composition in the gut [70].

Microbiota located in the caecum or colon (distal gut), such as Ruminococcaceae, produces energy and nutrients through the degradation of substrates such as non-starch polysaccharides to simple sugars using hydrolytic enzymes [8]. Faecalibacterium aids in the fermentation of these sugars resulting in the production of substances like short-chain fatty acids (SCFAs), i.e. butyrate and essential amino acids available for host consumption [87]. Production of butyrate in chickens is crucial for reducing chronic inflammation and relieving the severity of E. tenella infection [14]. The presence of SCFAs not only serves as the energy and carbon source for broilers, but also aids in the regulation of blood flow, which stimulates cell growth in the intestinal lining [16]. The degradation or fermentation of carbohydrates and polysaccharides releases easily accessible energy to other microorganisms, allowing further metabolic processes to occur [60].

\section{Normal gut anatomy and microbiota}

For chickens to effectively utilize end-products resulting from metabolic processes, a balanced microbial community is crucial to facilitate internal interaction between host and diet [86]. The chicken GIT is divided into sections consisting of diverse and unique microflora playing different metabolic functions; these sections include the crop, proventriculus, gizzard, small (jejunum and duodenum) and large intestines (ileum, caecum and colon) $[61,67,80]$. These sections harbour diverse communities of commensal, symbiotic and pathogenic microorganisms, e.g. crop, duodenum, and gizzard share almost similar microbiota composition (about 99\% Lactobacilli population) [24]. The bacterial composition and diversity within the GIT influence intestinal functions such as digestion and nutrient absorption. A balanced chicken GIT is predominated by Firmicutes, Tenericutes, Bacteroidetes, and Proteobacteria [8]. These commensal bacteria are located in the crop, with Lactobacillus exhibiting the highest diversity. They aid in the hydrolysis of starch and fermentation of lactate [10]. They are also present in high abundance in the ileum, facilitating nutrient absorption [66]. It has been observed that even though the composition of gut microbiota located in the ileum is more distinct and dominated by Lactobacillus, C. arthromitus, Enterococcus, and Clostridium, it is also less stable compared to other sections of the GIT (duodenum and the jejunum) [51, 60].

The caecum is known to have the most remarkable taxonomic diversity and shelter more diverse microflora, including anaerobes, e.g. Clostridium, Bacteroides, Proteobacteria, Actinobacteria and Firmicutes [67, 76, 95]. As broilers advance in age, caecal gut microbiota undergoes significant 


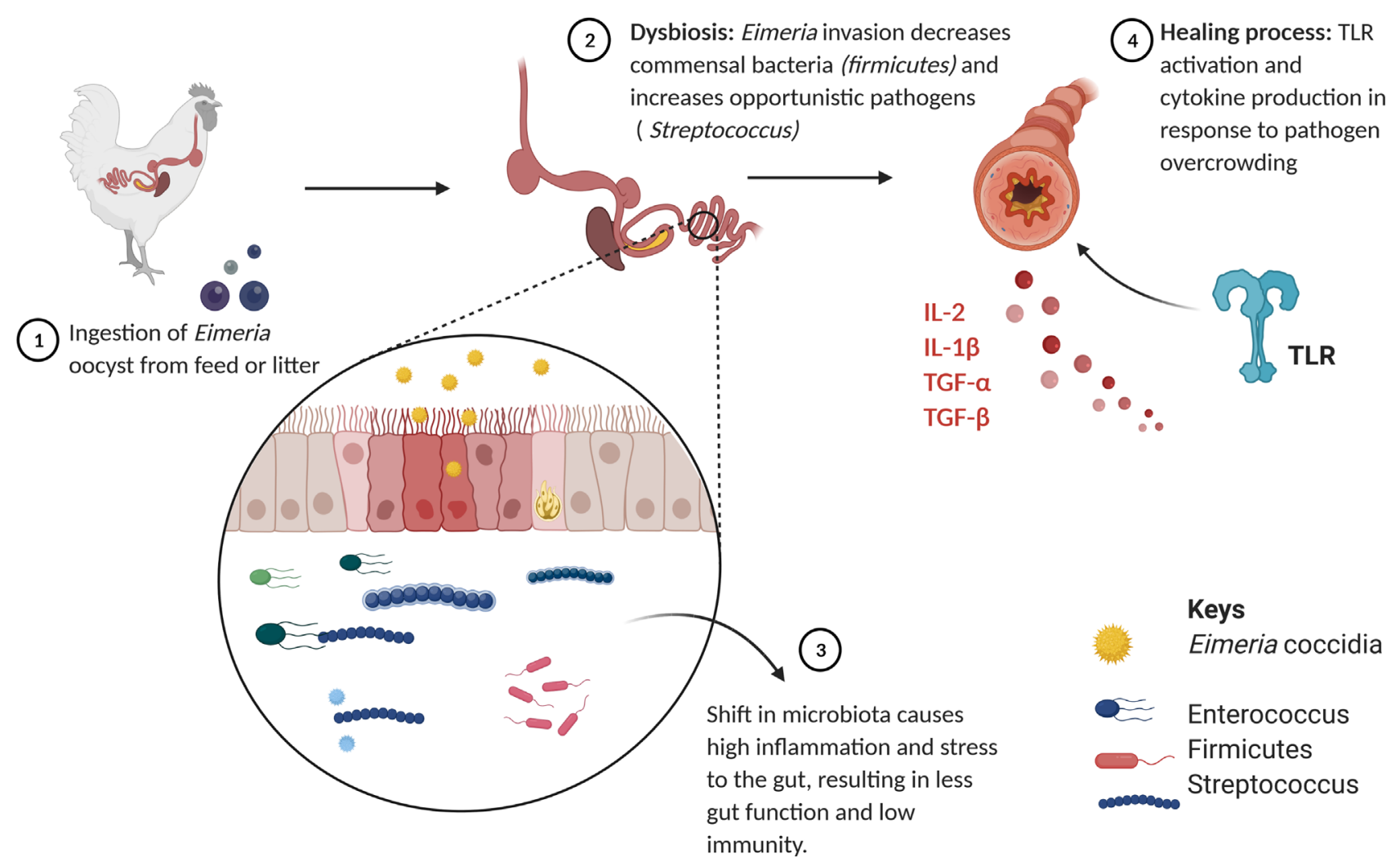

Figure 1. Impact of coccidiosis on bacterial species in the gastrointestinal tract of chickens.

changes resulting in a richer and stable microbial community [25]. These microbes play a crucial role in the fermentation and digestion of complex substrates such as cellulose, starch and other polysaccharides [81]. Microbial richness and diversity can be observed in the caecum during the second week, where most microbes from the phylum Proteobacteria are replaced and dominated by Firmicutes-Lachnospiraceae and Ruminococcaceae [40]. Previous research shows that changes in caecal microbial communities are likely due to microbe transfer through direct contact with the adult hen to offspring and chicks ingesting the mother's faecal material [80]. During the direct transfer, hens are significant donors of Bacteroidetes and Actinobacteria [40]. Development and changes in gut microbiota often compromise the immune system and health of chicks, leaving them susceptible to various bacterial and parasitic infections, e.g. Salmonella, C. perfringens, and Eimeria. Direct contact of offspring with older hens plays a vital role in microbial development and maturation regardless of environmental factors (feed and litter) [61]. Since microbiota becomes more diverse and stable as birds grow, changes observed in the gut, whether genetically transmitted by breeder birds or influenced by the environment, often result in a build-up of harmful bacteria which disrupts gut microbiota over beneficial ones [3, 78].

\section{Effect of coccidian (Eimeria) infection and pathogenicity in chicken gut microbiota}

Eimeria infection has a severe impact on broilers resulting in known physical symptoms such as depressed growth performance, decline in body weight gain, and low production
$[47,95]$. These clinical effects remain a leading contributor to economic losses, with the poultry industry suffering high losses due to treatment and chicken mortality. The external expression of these clinical symptoms is due to internal disruption of enterocytes and intestinal epithelial cells, causing unevenness in intestinal homeostasis and elevated susceptibility risk of the host to other diseases [29, 70]. During an E. tenella infection, the presence of severe intestinal epithelial injuries negatively impacts the proliferation and growth of resident bacteria [14]. Cytokines such as IL-17A, IL-10, IL-1 $\beta$ and IFN- $\gamma$ are secreted, providing pathways that favour proliferation and survival of pathogens [85]. Eimeria uses these pathways (Toll-like receptors-TLR-2 and TLR 6) to exploit the IL-10 mRNA production, invade the host immune system and complete its life cycle [68]. The highjacking of pathways by Eimeria poses a great threat to the health of chickens, affecting proper production and utilisation of nutrients.

Eimeria infection destroys the structure of caecal tissues and intestinal lining, causing a disturbance in the gut microbial community, known as dysbiosis [18]. Dysbiosis is exhibited by a significant fluctuation of beneficial bacteria, while harmful bacteria accumulate to the extent of becoming a potential threat to the host, causing an imbalance in host homeostasis [17]. An overview of Eimeria infection impact on bacterial species of the gut is shown in Figure 1. Zhou et al. [95] reported that E. tenella infection altered composition and diversity of caecal microbiota, significantly reducing Proteobacteria and Firmicutes (Enterococcus). It also causes shifted gut microbiota, reducing the caecal microbial diversity of chickens [87]. Eimeria infection affects the bird's ability to digest nutrients by reducing 
intestinal barrier function, causing bacterial translocation, affecting bacteria-dependent metabolic processes in the GIT [70]. Latorre et al. [41] reported that necrotic enteric diseases like coccidiosis affected the diversity and composition of the bacterial community in the GIT, reducing bacterial species such as Firmicutes, (mainly Ruminococcaceae) and SCFA-producing bacteria. Eimeria infection can decrease the frequency of immune-modulating bacteria (i.e. Candidatus savagella, Ruminococceae), while the abundance of bacteria (Clostridium spp.) that cause lesions and coccidia replication increases, causing severe damage to the mucosa [27]. The impact of coccidiosis bacterial species is summarised in Figure 1.

A study by Macdonald et al. [48] examining the effect of Eimeria infection (E. tenella) on caecal microbiome diversity revealed that Eimeria infection did not affect microbial alpha diversity but induced significant changes observed by severe lesion score damage in the caecum and reduction in the microbial population of Bacillales and Lactobacillales, suggesting that changes in abundance of the bacterial community may contribute to the severity of pathology and variation observed in tissue damage. Co-infections with Eimeria and C. perfringens also result in severe necrotic enteritis lesions with a significant increase of Clostridium sensu stricto 1, Escherichia, Shigella, and Weissella, and reduction in the Lactobacillus population located in the jejunum [10, 84]. Reduction of commensal bacteria such as Firmicutes (Lactobacillus) affects microbial diversity. It disrupts crucial metabolic processes that provide energy and carbon sources of the host [89]. These bacteria aid in converting glucose to lactic acid, lactate, acetic acid, ethanol and $\mathrm{CO}_{2}$, which serves as an energy powerhouse for the host [22]. Proteobacteria (Campylobacter) prevalence assists hydrogenases in hydrolysing indigestible sugars, namely; polysaccharides, oligosaccharides, and disaccharides aiding in the production of short chain fatty acids (SFCAs) [9]. Depletion of these bacteria due to E. tenella infection results in the build-up of complex compounds like uric acid and non-starch substrate indigestion. These compounds can be toxic to the host, hindering the production of amino acids essential for facilitating the production of SCFAs absorbed by enterocytes [16].

Some reports show that Eimeria infection alone has little to no effect on $\alpha$-diversity and caecal microbiota. However, co-infections with Eimeria, and other predisposing factors (C. perfringens) affecting the GIT can reshape or shift intestinal microbiota. Microbial shift results in loss of diversity and depletion of crucial gut microbiota, e.g. Lactobacillus, while favouring overgrowth of pathogenic bacterial strains (Clostridium, Salmonella and Weissella) that affect growth and health of the birds [28, 40, 50, 56, 93]. Supplementation of deficient bacteria to control Eimeria challenge is crucial, by the administration of natural dietary feeds/additives such as probiotics, prebiotics, and phytochemicals, which are able to improve function and the abundance of microbes, thus modulating gut microbiota.

\section{Dietary supplementation for manipulating the chicken gut microbiota}

Various strategies are currently being employed to combat the severity and spread of coccidiosis in chickens. Some of these control strategies include the use of live vaccines, therapeutic antimicrobial growth promoters (AGPs) and anticoccidial drugs (such as ionophore-based prophylactic drugs) produced from synthetic chemicals and fermentation methods [5, 34, 57]. The use of these control strategies in previous years has proved effective in controlling the spread of Eimeria infections; however, continuous exposure of animals to live vaccines and AGPs has been discouraged as it poses negative impacts on food animals and indirectly public health concerns to humans [88].

The use of live vaccines and AGPs (ionophores) hinders host colonisation by parasites through disruption of the parasite's replication cycle, interference of ion transfer, and other biochemical pathways in the plasma membrane of the parasite, leading to parasite death. Regardless of the effectiveness of these controls, continuous exposure of poultry to such treatments has resulted in the development of drug resistance and detection of traces of chemicals or compounds in poultry products (meat and eggs) as residuals that could potentially be harmful to humans when consumed, raising concerns and prompting calls for their discontinuation [5, 57, 64]. As a result, a diversion is observed in research to explore various antibioticindependent alternative strategies that are safe, antibiotic-free, and cost-effective to ameliorate coccidiosis in poultry, e.g. the use of probiotics, prebiotics, organic acids, phytobiotics and other natural additives, such as diet supplements in broiler feed [1, 23, 94]. Coccidiosis greatly impacts gut barrier function, which is crucial for optimal health and defence of host; hence, an ideal feed additive should promote efficient barrier function of the gut. Chen et al. [15] identified potential biomarkers (IL-8 and TGF- $\beta$ ) for barrier failure in broilers relevant for gut functioning in chickens. Administration of natural feeds such as probiotics should trigger cytokine production (IL-2, TGF- $\alpha$ ) to confront coccidiosis.

\section{Modulation of gut microbiota by probiotics against coccidial infection}

Probiotics are live, well-defined non-pathogenic culture of microorganisms ingested by the host, crucial in improving inhabitant intestinal microbiota. These are often bacteria or yeast extracts added to the broiler's diet to enhance production and animal health by improving the intestinal microbial balance [5]. These bacteria or extracts secrete crucial substances like bacteriocins and organic acids that contribute to health and homeostasis of the intestinal tract. They can protect against the invasion of pathogenic bacteria and coccidiosis by competitive exclusion [12, 28]. Investigating probiotics as potential alternative strategies to alleviate Eimeria challenge in broilers is still crucial following previous literature reporting on the benefits of using these natural additives. Probiotics have been reported to exhibit immunomodulation properties by manipulating and modifying gut microbiota, resulting in improved feed conversion ratio (FCR) and performance in broilers [5, 74]. Some of the bacteria used as probiotics are isolated from fermented milk, including Lactobacillus, Bacillus, Pediococcus, Saccharomyces cerevisiae and Enterococcus faecium [19]. $\mathrm{Li}$ et al. [46] reported effective response observed in broilers upon administration of Lactobacillus-based probiotics (L. acidophilus), which boosted inflammatory response through 
production of acidophilin and modulated the immune responses (innate and acquired) of poultry, providing a favourable environment for beneficial bacteria [24].

During Eimeria infection, the parasites replicate and adhere to receptors located in the intestine epithelial surface. Intestinal compatible probiotic bacteria challenge Eimeria parasites in adhering to the intestinal mucosa and absorb receptors in the epithelial cells, preventing invasion [33]. This attachment hinders the perforation and secretion of Eimeria sporozoites into the intestinal mucosa, further resulting in reduced proliferation and oocyst shedding [58]. This regulates host intestinal permeability, improves barrier functions of the gut, and balances host gut microbiota [31]. Eimeria infection affects intestinal permeability, so the administration of feed additive like probiotics could promote the balance of gut microbiota and regulate the barrier function to prevent the entry of foreign microorganisms in the GIT.

Probiotics improve beneficial bacteria's ability to adhere to the GIT, enabling them to occupy most of the tract while inhibiting the growth of pathogenic bacteria and parasites [84]. Probiotics employ other competitive exclusion modes of action to reduce parasite colonisation, including competition for sites of attachments, co-aggregation with pathogens and certain gut microbes and production of antimicrobial compounds that stimulate the immune system, e.g. lactic acid, hydrogen peroxide and bacteriocins $[2,38]$. These mechanisms promote animal growth and balance in gut health through microbial abundance in the GIT [69]. Competitive exclusion exhibited by probiotics against Eimeria species results in improved intestinal health through gut maturation and improved integrity, leading to improved feed digestion and absorption, hence higher body weight gain is often observed in chickens after probiotic treatment [65]. Alagawany et al. [2] reviewed the modes of actions exhibited by probiotics to hinder invasion and proliferation of pathogens such as Eimeria and Salmonella. The mechanism employed by probiotics involves the production of antibacterial substances that play a role competitively excluding unwanted and harmful bacteria from entering the gut $[2,38]$. Probiotics enhance host immune responses by regulating T-helper cells, conferring improved protection against the Eimeria challenge [26]. Research has revealed probiotics as a promising alternative to antibiotics; however, probiotic incorporation is highly dependent on chicken diet (optimal dose) and selection of appropriate strains of probiotic microbes to exhibit effective action against specific pathogens [18].

Probiotics are also crucial for stimulating the proliferation of the intestinal epithelium, which regulates the mucosal barrier by mucin in the intestinal wall of broilers, lowering bacterial diversity $[19,26]$. The use of probiotics such as Bacillus subtilis as dietary supplementation modifies gut microbiota composition by improving growth performance and nutrient digestion in broilers through an increased abundance of Bacteroidetes and other commensal bacteria, such as Ruminococcus [46]. Similar findings were reported by Wang et al. [84], where B. subtilis was confirmed to reduce microbial diversity in the caecum by altering microbial community and elevating predominant species. The abundance of these bacteria aids in the breakdown of indigestible fibres, releasing substances such as butyrate and SCFAs, which are essential for extra nutrients and energy for broilers [24, 44]. Probiotics mainly target microorganisms in the small intestines, where peak nutrient absorption is observed [84]. The presence of probiotics (i.e., Lactobacillus) in different sites in the GIT increases nutrient utilisation, hindering intestinal colonisation and intestinal lesions caused by E. tenella invasion [65]. In evaluating the immune effect of infection in broilers, Awais et al. [5] revealed that Lactobacillus and Saccharomyces-based probiotics enhanced immunological and performance capabilities in broilers challenged with Eimeria. This property enables probiotics to protect against Eimeria infections, thereby reducing coccidiosis prevalence.

Lactobacillus-based probiotics have been reported to exert vital anticoccidial properties against $E$. tenella infections $[5,14]$. These probiotics exhibit growth-promoting effects that promote enhanced cellular and humoral immune responses, restricting the invasion of the GIT by Eimeria spp. [44]. They also act as antagonists by producing cytokines and local antibodies (immunoglobulin A-IgA), which are crucial for confronting coccidiosis, i.e. interleukin-2 (IL-2), interferon- $\gamma$ (IFN- $\gamma$ ) and IL-6, and stimulate local cell-mediated immunity inhibiting invasion by $E$. acervulina [7, 24, 26, 74]. Cytokines are natural proteins essential for stimulation and regulation of immunity against infectious diseases. These substances also hinder the secretion of siderophores, restricting iron availability to facilitate parasite invasion [5]. Probiotics also protect against Eimeria by stimulating mucosal immunity and reducing oocyst shedding [45].

Chen et al. [13] previously showed that a probiotic mixture containing four strains of lactic acid bacteria (L. acidophilus, L. fermentum, L. planetarium, and E. faecium) was effective in reducing intestinal ulcers observed in broilers due to E. tenella infection, and significantly influenced the level of expression of specific genes crucial for inflammation, e.g. cytokines [26]. Lactobacillus-based probiotics reduced levels of caecal gene expression of cytokines favouring inflammation (e.g. interleukin (IL)-1 $\beta$ and IL-6) and interferon (IFN)- $\gamma$, while anti-inflammation cytokine, i.e. anti-IL-10, was increased [4, 13]. Probiotics' capability to exert stimulating effects on the immune system, while altering the gut microbiota profile [50], cement their consideration as suitable candidates to control coccidiosis. More research has been done to determine the effectiveness of other probiotic microflora against coccidiosis. Enterococcus faecium increased the abundance of beneficial bacteria, while also modulating the composition of intestinal microflora. Enterococcus faecium and Pediococcus supplementation in broilers infected with $E$. acervulina exhibited immunomodulatory effects through increased production of cytokines aiding in the modulation of anti-inflammatory cytokines and other immune mediators, including IL-1 $\beta$, IL-6, IL-10, and IFN- $\gamma$, reducing the severity of intestinal lesions caused by the infection [43, 77, 87]. Although in-depth information on anticoccidial properties of some probiotics has been reported, efforts in exploring other microflora in the gut with potential to induce protection against necrotic enteritis infectious diseases is still required.

Even though probiotics effectively control coccidiosis, research has revealed that simultaneous inclusion of prebiotics with probiotics as poultry feed additives can enhance the 
Table 1. Different phytochemical additives against coccidiosis in poultry.

\begin{tabular}{ll}
\hline Phytochemical & \multicolumn{1}{c}{ Effect on gut health and immune response } \\
\hline Areca Nut (Areca Catechu L.) & - Reduced caecal lesion scores. \\
& - Enhanced immunity through the production of interleukin 2 (IL-2) [83] \\
Bidens Pilosa & - Disrupted life cycle of E. tenella $[12,91]$ \\
& - Enhanced T-cell mediated immunity [14, 92] \\
Carvacrol & - Increased growth performance and intestinal barrier function [20] \\
& - Altered gut physiology \\
& - Interference with the life cycle of E. tenella by destroying sporozoite membrane [42] \\
Curcumin & - Maintenance of gut integrity [88] \\
& - Interference with life cycle by inhibiting E. tenella sporozoites [11] \\
Cinnamaldehyde & - Improved chicken growth performance and altered caecal microbiota composition [90] \\
Garlic (Allium Sativum) & - Increased resistance to experimental Eimeria acervulina infection [62, 71] \\
\end{tabular}

viability of probiotic microorganisms through a synergistic effect [14, 44]. Pineda-Quiroga et al. [59] showed that supplementation with synbiotics in the broiler diet conferred modulatory effect on microbiota, while enhancing various pathways (i.e. starch and sucrose metabolism) without interfering with the biological role of caecal microbiota compared to when pre- and probiotics are administered independently. Similarly, the combination of pro- and prebiotics allows prebiotics to activate probiotics for effective modulation of the metabolic response in the gut against infections, while maintaining gut microbiota integrity and inhibition of potential pathogens present in the digestive system [73]. Muthamilselvan et al. [53] further reviewed anticoccidial action of herbal remedies against coccidiosis, noting close interactions between prebiotics and probiotic microorganisms inhabiting the gut. Prebiotics are non-digestible feed additives that contain natural dietary fibres such as fructooligosaccharides (FOS), inulin, $\beta$-glucans, and mannooligosaccharides (MOS), which are crucial in inducing development or activity of commensal bacteria in the gut, excluding invasion of the harmful pathogens $[23,55,56]$. Accumulation of gut probiotics mediated by prebiotics suppresses pathogens and significant improvement of poultry's immune responses [88]. Even though Eimeria infections drastically reduce majoring of commensal bacteria, studies have found a mild increase of some Firmicutes (Ruminococcaceae) in infected chickens after E. tenella infection [14]. Microbe recovery after infection allows fast restoration of GIT mucosa, maintaining gut homeostasis.

\section{Stimulation and stabilisation of gut microbiota through phytobiotics}

The use of plant-based phytochemicals has been associated with their antimicrobial and antiparasitic properties that enhance protective immunity in livestock infected by coccidiosis and other necrotic enteritis diseases [32]. Phytobiotics include various products synthesized and extracted from plants such as herbs, essential oils, and oleoresins [52]. Supplementation of these natural products as animal feeds has been favoured because they are safe, cheap, readily available in nature and effective against various diseases $[1,57]$. Some of these natural foods and herbs improve immunity and animal resistance to disease. The mechanisms of action utilized by phytochemicals in controlling infections include interference with the life cycle of $E$. tenella by hindering oocyst sporulation, invasion of sporozoites and the maturation of schizonts [93]. They have been reported to impair Eimeria spp. in early development stages by reducing cell wall degradation and inducing oxidative stress, preventing invasion $[20,53]$. Similar findings were reported by Jiao et al. [35] where administration of Artemisia annua (A. annua) and Artemisinin was confirmed to interfere with the Eimeria life cycle by producing reactive oxygen species that are effective in inhibition of oocyst sporulation and formation of the parasite cell wall.

Research done to date on phytonutrients such as Capsicum annuum (pepper), Curcuma longa (turmeric), Lentinus edodes (shiitake mushroom), and Carthamus tinctorius (safflower) revealed promising inhibitory properties and defence mechanisms against infections in chickens, promoting enhanced gut health and immune system $[39,82]$. There is a wide range of commercialised plant-derived compounds available, and their effect on chicken health thus far has been promising. Some phytochemicals and their effect on immune response and gut health are presented in Table 1. Administration of phytochemicals in chickens challenged with Eimeria alters and stabilises the intestinal microbiota by shifting the composition of beneficial bacteria and reducing microbial metabolites in the gut, improving gut health [90]. Kim et al. [39] further confirmed that the administration of capsicum and curcumin longa oleoresins affected the microbial population by increasing abundance of Lactobacillus and operational taxonomic units, while reducing the population of Selenihalanaerobacter in two chicken breeds (Ross and Cobb). Cinnamaldehyde and Oleoresins (Capsicum and Turmeric) have been reported to regulate host immunity against $E$. tenella through elevation of T helper cells and cytokines (FN- $\gamma$ and IL-6) and body weight gain in poultry [42]. A combination of curcuma and capsicum exert synergistic effects against coccidiosis by enhancing innate immunity. The mechanisms exhibited by phytobiotics on the regulation of the life cycle of Eimeria and growth regulation of gut bacteria make phytochemicals best suited for supplementation in the poultry diet at an early stage of development.

Similar results were found by Chowdhurya et al. (2018) when evaluating the effect of three essential oils, i.e. cinnamon 
bark oil (CNO), clove bud oil (CLO), and ajwain seed oil (AJO), where the abundance of Escherichia coli in pre-caecal contents decreased in groups given $\mathrm{CNO}$, but did not affect Lactobacillus spp. in any diets. Based on a range of activities exerted by phytobiotics in chickens, it can be suggested that the primary mode of action for most phytobiotics focuses on altering the gut environment and intestinal morphology, providing protection and enhanced resistance to infections when fed to healthy poultry $[32,36]$. From the findings mentioned above, the simultaneous use of feed additives may be beneficial to the host by providing different functions, such as maintaining chicken gut health and targeting different aspects of animal physiology to boost antimicrobial activity against diseases. Research has also shown that combined supplementation of phytochemicals exerts boosted anticoccidial effects in broilers compared to when administered on their own [20, 72].

\section{Conclusion and future recommendations}

Coherent interaction between gut microbiota and the host is crucial for the normal functioning and health of poultry. Presence of a coccidian infection (i.e. Eimeria or Isospora) in chickens compromises gut microbiota resulting in an imbalance of the microbial communities, affecting their active role within a host. Supplementation with natural alternatives such as probiotics and phytochemicals remains the most favoured strategy currently to combat coccidiosis, without the effect of drug resistances threatening biosecurity. Manipulation of gut microbiota by introducing natural additives at an early stage of development may improve the immune system of chickens against Eimeria infections. Since these additives are chemicals and strains fermented by bacteria, they can persist in the GIT as a beneficial gut microbiome for a long time, without posing any adverse effect on broilers. Thus, the use of natural alternatives (feed additives) is recommended for fast GIT restoration and modulation of gut microbiota in response to coccidian challenges, while improving growth performance and overall health of broilers. Significant progress has been made in discovering alternative strategies to combat coccidiosis; however, further studies focusing on joint interaction of feed additive with host gut microbiota towards Eimeria challenge are still required to provide efficacious treatment measures.

Acknowledgements. The authors wish to thank the National Research Foundation (NRF) of South Africa for financial support (Thuthuka Grant number: 112768 and S\&F-Innovation Masters scholarship grant number: 123320).

\section{Author contributions}

T Madlala wrote the manuscript. M Okpeku and MA Adeleke supervised the work and corrected the manuscript. All authors approved the manuscript.

\section{Declaration of conflict of interest}

The authors declare that they have no conflict of interest.

\section{References}

1. Abbas A, Iqbal Z, Abbas RZ, Khan MK, Khan JA, Hussain K, Mahmood MS, Rizwan HM. 2017. Immunomodulatory effects of Camellia sinensis against coccidiosis in chickens. Journal of Animal and Plant Sciences, 27, 415-421.

2. Alagawany M, El-Hack MEA, Farag MR, Sachan S, Karthik K, Dhama K. 2018. The use of probiotics as eco-friendly alternatives for antibiotics in poultry nutrition. Environmental Science and Pollution Research, 25, 10611-10618.

3. Antonissen G, Eeckhaut V, Van Driessche K, Onrust L, Haesebrouck F, Ducatelle R, Moore RJ, Van Immerseel F. 2016. Microbial shifts associated with necrotic enteritis. Avian Pathology, 45, 308-312.

4. Arendt MK, Sand JM, Marcone TM, Cook ME. 2016. Interleukin-10 neutralizing antibody for detection of intestinal luminal levels and as a dietary additive in Eimeria challenged broiler chicks. Poultry Science, 95, 430-438.

5. Awais MM, Jamal MA, Akhtar M, Hameed MR, Anwar MI, Ullah MI. 2019. Immunomodulatory and ameliorative effects of Lactobacillus and Saccharomyces based probiotics on pathological effects of eimeriasis in broilers. Microbial Pathogenesis, 126, 101-108.

6. Baldwin S, Hughes RJ, Van Hao TT, Moore RJ, Stanley D. 2018. At-hatch administration of probiotic to chickens can introduce beneficial changes in gut microbiota. PloS One, 13.

7. Behnamifar AR, Rahimi S, Kiaei MM, Fayazi H. 2019. Comparison of the effect of probiotic, prebiotic, salinomycin and vaccine in control of coccidiosis in broiler chickens. Iranian Journal of Veterinary Research, 20, 51-54.

8. Borda-Molina D, Seifert J, Camarinha-Silva A. 2018. Current Perspectives of the chicken gastrointestinal tract and its microbiome. Computational and Structural Biotechnology Journal, 16, 131-139.

9. Bortoluzzi C, Barbosa JGM, Pereira R, Fagundes NS, Rafael JM, Menten JFM. 2018. Autolyzed Yeast (Saccharomyces cerevisiae) Supplementation improves performance while modulating the intestinal immune-system and microbiology of broiler chickens. Frontiers in Sustainable Food Systems, 2, 85.

10. Bortoluzzi C, Vieira BS, Hofacre C, Applegate TJ. 2019. Effect of different challenge models to induce necrotic enteritis on the growth performance and intestinal microbiota of broiler chickens. Poultry Science, 98, 2800-2812.

11. Burt SA, Tersteeg-Zijderveld MHG, Jongerius-Gortemaker BGM, Vervelde L, Vernooij JCM. 2013. In vitro inhibition of Eimeria tenella invasion of epithelial cells by phytochemicals. Veterinary Parasitology, 191(3-4), 374-378.

12. Chang CL, Chung CY, Kuo CH, Kuo TF, Yang CW, Yang WC. 2016. Beneficial effect of Bidens pilosa on body weight gain, food conversion ratio, gut bacteria and coccidiosis in chickens. PLoS One, 11(1), e0146141.

13. Chen CY, Chuang LT, Chiang YC, Li Lin C, Lien YY, Tsen HY. 2016. Use of a probiotic to ameliorate the growth rate and the inflammation of broiler chickens caused by Eimeria tenella Infection. Journal of Animal Research and Nutrition, 1, 10.

14. Chen HL, Zhao XY, Zhao GX, Huang HB, Li HR, Shi CW, Yang WT, Jiang YL, Wang JZ, Ye LP, Zhao Q, Wang CF, Yang GL. 2020. Dissection of the caecal microbial community in chickens after Eimeria tenella infection. Parasites \& Vectors, $13,56$.

15. Chen J, Tellez G, Richards JD, Escobar J. 2015. Identification of potential biomarkers for gut barrier failure in broiler chickens. Frontiers in Veterinary Science, 2, 14. 
16. Clavijo V, Flórez MJV. 2017. The gastrointestinal microbiome and its association with the control of pathogens in broiler chicken production: a review. Poultry Science, 97, 1006-1021.

17. Cui N, Wang X, Wang Q, Li H, Wang F, Zhao X. 2017. Effect of dual infection with Eimeria tenella and subgroup J avian leukosis virus on the caecal microbiome in specificpathogen-free chicks. Frontiers in Veterinary Science, 4, 177.

18. Ducatelle R, Eeckhaut V, Haesebrouck F, Van Immerseel F. 2015. A review on prebiotics and probiotics for the control of dysbiosis: present status and future perspectives. Animal, 9, 43-48.

19. Erdoğmuş SZ, Gülmez N, Findik A, Şah H, Gülmez M. 2019. Efficacy of probiotics on health status and growth performance of Eimeria tenella infected broiler chickens. Kafkas Universitesi Veteriner Fakultesi Dergisi, 25(3), 311-320.

20. Felici M, Tugnoli B, Ghiselli F, Massi P, Tosi G, Fiorentini L, Piva A, Grilli E. 2020. In vitro anticoccidial activity of thymol, carvacrol, and saponins. Poultry Science, 99(11), 5350-5355.

21. Fetterer RH, Allen PC. 2001. Eimeria tenella infection in chickens: effect on plasma and muscle 3-methylhistidine. Poultry Science, 80(11), 1549-1553.

22. Forte C, Manuali E, Abbate Y, Papa P, Vieceli L, Tentellini M, Trabalza-Marinucci M, Moscati L. 2018. Dietary Lactobacillus acidophilus positively influences growth performance, gut morphology, and gut microbiology in rurally reared chickens. Poultry Science, 97(3), 930-936.

23. Gadde U, Kim WH, Oh ST, Lillehoj HS. 2017. Alternatives to antibiotics for maximizing growth performance and feed efficiency in poultry: a review. Animal Health Research Reviews, $18,26-45$.

24. Gharib-Naseri K, Kheravii SK, Keerqin C, Morgan N, Swick RA, Choct M, Wu SB. 2019. Two different Clostridium perfringens strains produce different levels of necrotic enteritis in broiler chickens. Poultry Science, 98(12), 6422-6432.

25. Gong Y, Yang H, Wang X, Xia WLW, Xiao Y, Zou X. 2019. Early intervention with caecal fermentation broth regulates the colonization and development of gut microbiota in broiler chickens. Frontiers in Microbiology, 10, 1422.

26. Gu X, Zhang J, Li J, Wang Z, Feng J, Li J, Pan K, Ni X, Zeng D, Jing B, Zhang D. 2019. Effects of Bacillus cereus PAS38 on immune-related differentially expressed genes of spleen in broilers. Probiotics and Antimicrobial Proteins, 12(2), 425-438.

27. Hauck R. 2017. Interactions between parasites and the bacterial microbiota of chickens. Avian Diseases, 61, 428-436.

28. Hernandez-Patlan D, Solis-Cruz B, Pontin KP, HernandezVelasco X, Merino-Guzman R, Adhikari B, Lopez-Arellano R, Kwon YM, Hargis BM, Arreguin-Nava MA, Tellez-Isaias G, Latorre JD. 2019. Impact of a Bacillus direct-fed microbial on growth performance, intestinal barrier integrity, necrotic enteritis lesions, and ileal microbiota in broiler chickens using a laboratory challenge model. Frontiers in Veterinary Science, 6, 108.

29. Hessenberger S, Schatzmayr G, Teichmann K. 2016. In vitro inhibition of Eimeria tenella sporozoite invasion into host cells by probiotics. Veterinary Parasitology, 229, 93-98.

30. Huang G, Zhang S, Zhou C, Tang X, Li C, Wang C, Tang X, Suo J, Jia Y, El-Ashram S, Yu ZCJ, Gupta N, Suo X, Liua X. 2018. Influence of Eimeria falciformis infection on gut microbiota and metabolic pathways in mice. Infection and Immunity, 86(5), e00073-18.

31. Huyghebaert G, Ducatelle R, Van Immerseel F. 2011. An update on alternatives to antimicrobial growth promoters for broilers. Veterinary Journal, 187, 182-188.

32. Idris M, Abbas R, Masood S, Rehman T, Farooq U, Babar W, Hussan R, Raza A, Riaz U. 2017. The potential of antioxidant rich essential oils against avian coccidiosis. World's Poultry Science Journal, 73(1), 89-104.
33. Jarujareet W, Shigenoki Y, Taira K, Ooi HK. 2018. Eimeria tenella oocyst excretion and riboflavin supplement in infected chicken. Journal of Veterinary Medical Science, 80(9), 1392-1394.

34. Jenkins MC, Parker CC, O'Brien CN, Ritter D. 2019. Viable Eimeria oocysts in poultry house litter at the time of chick placement. Poultry Science, 98(8), 3176-3180.

35. Jiao J, Yang Y, Liu M, Li J, Cui Y, Yin S, Tao J. 2018. Artemisinin and Artemisia annua leaves alleviate Eimeria tenella infection by facilitating apoptosis of host cells and suppressing inflammatory response. Veterinary Parasitology, 254, 172-177.

36. Jitviriyanon $\mathrm{S}$, Phanthong $\mathrm{P}$, Lomarat $\mathrm{P}$, Bunyapraphatsara $\mathrm{N}$, Porntrakulpipat S, Paraksa N. 2016. In vitro study of anticoccidial activity of essential oils from indigenous plants against Eimeria tenella. Veterinary Parasitology, 228, 96-102.

37. Kers JG, Velkers FC, Fischer E, Hermes G, Stegeman JA, Smidt H. 2018. Host and environmental factors affecting the intestinal microbiota in chickens. Frontiers in Microbiology, 9, 235.

38. Khan M, Anjum AA, Nawaz M, Awan AR, Ali MA. 2019. Effect of newly characterized probiotic Lactobacilli on weight gain, immunomodulation and gut microbiota of Campylobacter jejuni challenged broiler chicken. Pakistan Veterinary Journal, 39, 473-478.

39. Kim JE, Lillehoj HS, Hong YH, Kim GB, Lee SH, Lillehoj EP, Bravo DM. 2015. Dietary Capsicum and Curcuma longa oleoresins increase intestinal microbiome and necrotic enteritis in three commercial broiler breeds. Research in Veterinary Science, 102, 150-158.

40. Kubasova T, Kollarcikova M, Crhanova M, Karasova D, Cejkova D, Sebkova A, Matiasovicova J, Faldynova M, Pokorna A, Cizek A, Rychlik I. 2019. Contact with adult hen affects development of caecal microbiota in newly hatched chicks. PloS One, 14, e0212446.

41. Latorre JD, Adhikari B, Park SH, Teague KD, Graham LE, Mahaffey BD, Baxter MFA, Hernandez-Velasco X, Kwon YM, Ricke SC, Bielke LR, Hargis BM, Tellez G. 2018. Evaluation of the epithelial barrier function and ileal microbiome in an established necrotic enteritis challenge model in broiler chickens. Frontiers in Veterinary Science, 5, 199

42. Lee JW, Kim DH, Kim YB, Jeong SB, Oh ST, Cho SY, Lee KW. 2020. Dietary encapsulated essential oils improve production performance of coccidiosis-vaccine-challenged broiler chickens. Animals, 10(3), 481.

43. Lee KW, Lillehoj HS, Jang SI, Lee SH. 2014. Effects of salinomycin and Bacillus subtilis on growth performance and immune responses in broiler chickens. Research in Veterinary Science, 97(2), 304-308.

44. Leung H, Yitbarek A, Snyder R, Patterson R, Barta JR, Karrow N, Kiarie E. 2018. Responses of broiler chickens to Eimeria challenge when fed a nucleotide-rich yeast extract. Poultry Science, 98(4), 1622-1633.

45. Leung H. 2018. The effects of a nucleotide rich yeast extract on growth performance, gastrointestinal ecology and immune system in broiler chickens challenged with Eimeria. Masters, University of Guelph, Guelph, Ontario, Canada, 120 p.

46. Li Z, Wang W, Liu D, Guo Y. 2017. Effects of Lactobacillus acidophilus on gut microbiota composition in broilers challenged with Clostridium perfringens. PloS One, 12, e0188634.

47. Liu J, Liu L, Li L, Tian D, Li W, Xu L, Yan R, Li X, Song X. 2018. Protective immunity induced by Eimeria common antigen 14-3-3 against Eimeria tenella, Eimeria acervulina and Eimeria maxima. BMC Veterinary Research, 14(1), 337.

48. Macdonald SE, Nolan MJ, Harman K, Boulton K, Hume DA, Tomley FM, Stabler RA, Blake DP. 2017. Effects of Eimeria tenella infection on chicken caecal microbiome diversity, exploring variation associated with severity of pathology. PloS One, 12(9), e0184890. 
49. Macdonald SE, van Diemen PM, Martineau H, Stevens MP, Tomley FM, Stabler RA, Blake DP. 2019. Impact of Eimeria tenella coinfection on Campylobacter jejuni colonization of the chicken. Infection and Immunity, 87(2), e00772-18.

50. Moore RJ. 2016. Necrotic enteritis predisposing factors in broiler chickens. Avian Pathology, 45(3), 275-281.

51. Mohd Shaufi MA, Sieo CC, Chong CW, Gan HM, Ho YW. 2015. Deciphering chicken gut microbial dynamics based on high-throughput 16S rRNA metagenomics analyses. Gut Pathogens, 7(1), 1-12.

52. Mohammadi Gheisar M, Kim IH. 2017. Phytobiotics in poultry and swine nutrition - a review. Italian Journal of Animal Science, 17(1), 92-99.

53. Muthamilselvan T, Kuo TF, Wu YC, Yang WC. 2016. Herbal remedies for coccidiosis control: A review of plants, compounds, and anticoccidial actions. Evidence Based Complementary and Alternative Medicine, 2016, 2016.

54. Nabian S, Arabkhazaeli F, Seifouri P, Farahani A. 2018. Morphometric analysis of the intestine in experimental coccidiosis in broilers treated with anticoccidial drugs. Iranian Journal of Parasitology, 13(3), 493-499.

55. Nopvichai C, Charoenwongpaiboon $\mathrm{T}$, Luengluepunya N, Ito K, Muanprasat C, Pichyangkura R. 2019. Production and purification of mannan oligosaccharide with epithelial tight junction enhancing activity. PeerJ, 7, e7206.

56. Ocejo M, Oporto B, Hurtado A. 2019. 16S rRNA amplicon sequencing characterization of caecal microbiome composition of broilers and free-range slow-growing chickens throughout their productive lifespan. Scientific Reports, 9, 1-14.

57. Oh S, Gadde UD, Bravo D, Lillehoj EP, Lillehoj HS. 2018. Growth-promoting and antioxidant effects of magnolia bark extract in chickens uninfected or co-infected with Clostridium perfringens and Eimeria maxima as an experimental model of necrotic enteritis. Current Developments in Nutritions, 2(4), nzy009.

58. Oyewole O, Maria C, Tope P, Funmi O. 2018. In vitro study of potential probiotic lactic acid bacteria isolated from the gut of chickens in Abeokuta, Nigeria. Alexandria Journal of Veterinary Sciences, 58(1), 73-84.

59. Pineda-Quiroga C, Borda-Molina D, Chaves-Moreno D, Ruiz R, Atxaerandio R, Camarinha-Silva A, García-Rodríguez A. 2019. Microbial and functional profile of the ceca from laying hens affected by feeding prebiotics, probiotics, and synbiotics. Microorganisms, 7(5), 123.

60. Pourabedin M, Guan L, Zhao X. 2015. Xylo-oligosaccharides and virginiamycin differentially modulate gut microbial composition in chickens. Microbiome, 3, 1-12.

61. Pourabedin M, Zhao X. 2015. Prebiotics and gut microbiota in chickens. FEMS Microbiology Letters, 362, fnv122.

62. Pourali M, Kermanshahi H, Golian A, Ramzi GR, Soukhtanloo M. 2014. Antioxidant and anticoccidial effects of garlic powder and sulfur amino acids on Eimeria-infected and uninfected broiler chickens. Iranian Journal of Veterinary Research, 15, 227-232.

63. Quiroz-Castañeda RE, Dantán-González E. 2015. Control of avian coccidiosis: future and present natural alternatives. BioMed Research International, 2015, 430610.

64. Reis JH, Gebert RR, Barreta M, Baldissera MD, Dos Santos ID, Wagner R, Campigotto G, Jaguezeski AM, Gris A, de Lima JLF, Mendes RE, Fracasso M, Boiago MM, Stefani LM, Dos Santos DS, Robazza WS, Da Silva AS. 2018. Effects of phytogenic feed additive based on thymol, carvacrol and cinnamaldehyde on body weight, blood parameters and environmental bacteria in broilers chickens. Microbial Pathogenesis, $125,168-176$
65. Royan M. 2019. A review on the lactic acid bacteria probiotic in the control of coccidiosis, Campylobacteriosis, and Salmonellosis in broiler chickens. Iranian Journal of Applied Animal Science, 9(1), 1-8.

66. Rodriguez-Sanchez R, Tres A, Sala R, Guardiola F, Barroeta AC. 2019. Evolution of lipid classes and fatty acid digestibility along the gastrointestinal tract of broiler chickens fed different fat sources at different ages. Poultry Science, 98, 1341-1353.

67. Rubio LA. 2019. Possibilities of early life programming in broiler chickens via intestinal microbiota modulation. Poultry Science, 98, 695-706.

68. Sand JM, Arendt MK, Repasy A, Deniz G, Cook ME. 2016. Oral antibody to interleukin-10 reduces growth rate depression due to Eimeria spp. infection in broiler chickens. Poultry Science, 95(2), 439-446.

69. Sethiya NK. 2016. Review on natural growth promoters available for improving gut health of poultry: an alternative to antibiotic growth promoters. Asian Journal of Poultry Science, $10,1-29$.

70. Shang Y, Kumar S, Oakley B, Kim WK. 2018. Chicken gut microbiota: importance and detection technology. Frontiers in Veterinary Science, 5, 254.

71. Sheoran N, Kumar R, Kumar AA, Batra K, Sihag S, Maan S, Maan NS. 2017. Nutrigenomic evaluation of garlic (Allium sativum) and holy basil (Ocimum sanctum) leaf powder supplementation on growth performance and immune characteristics in broilers. Veterinary World, 10, 121-129.

72. Sidiropoulou E, Skoufos I, Marugan-Hernandez V, Giannenas I, Bonos E, Aguiar-Martins K, Lazari D, Blake DP, Tzora A. 2020. In vitro anticoccidial study of Oregano and garlic essential oils and effects on growth performance, fecal oocyst output, and intestinal microbiota in vivo. Frontiers in Veterinary Science, 7, 420.

73. Slizewska K, Markowiak P, Zbikowski A, Szeleszczuk P. 2019. Effects of synbiotics on the gut microbiota, blood and rearing parameters of chickens. FEMS Microbiology Letters, 366(11), fnz116.

74. Smialek M, Burchardt S, Koncicki A. 2018. The influence of probiotic supplementation in broiler chickens on population and carcass contamination with Campylobacter spp. - Field study. Research in Veterinary Science, 118, 312-316.

75. Song X, Gao Y, Xu L, Yan R, Li X. 2015. Partial protection against four species of chicken coccidia induced by multivalent subunit vaccine. Veterinary Parasitology, 212, 80-85.

76. Stanley D, Wu SB, Rodgers N, Swick RA, Moore RJ. 2014. Differential responses of caecal microbiota to fishmeal, Eimeria and Clostridium perfringens in a necrotic enteritis challenge model in chickens. PloS One, 9, e104739.

77. Tarasova E, Yermolenko E, Donets V, Sundukova Z, Bochkareva A, Borshev I, Suvorova M, Ilyasov I, Simanenkov V, Suvorov AN. 2010. The influence of probiotic Enterococcus faecium strain L5 on the microbiota and cytokines expression in rats with dysbiosis induced by antibiotics. Beneficial Microbes, $1,265-270$.

78. Tilocca B, Witzig M, Rodehutscord M, Seifert J. 2016. Variations of phosphorous accessibility causing changes in microbiome functions in the gastrointestinal tract of chickens. PloS One, 11, e0164735.

79. Tsukahara T, Inoue R, Nakayama K, Inatomi T. 2018. Inclusion of Bacillus amyloliquefaciens strain TOA5001 in the diet of broilers suppresses the symptoms of coccidiosis by modulating intestinal microbiota. Animal Science Journal, 89, 679-687.

80. Turk DE. 1982. The anatomy of the avian digestive tract as related to feed utilization. Poultry Science, 61(7), 1225-1244. 
81. Vieira AM, Soratto T, Cardinal KM, Wagner G, Hauptli L, Lima A, Dahlke F, Peres Netto D, Moraes PO, Ribeiro A. 2020. Modulation of the intestinal microbiota of broilers supplemented with monensin or functional oils in response to challenge by Eimeria spp. PloS One, 15(8), e0237118.

82. Wagle BR, Upadhyay A, Upadhyaya I, Shrestha S, Arsi K, Liyanage R, Venkitanarayanan K, Donoghue DJ, Donoghue AM. 2019. Trans-cinnamaldehyde, eugenol and carvacrol reduce Campylobacter jejuni biofilms and modulate expression of select genes and proteins. Frontiers in Microbiology, 10, 1837.

83. Wang D, Zhou L, Li W, Zhou H, Hou G. 2018. Anticoccidial effects of areca nut (Areca catechu L.) extract on broiler chicks experimentally infected with Eimeria tenella. Experimental Parasitology, 184, 16-21.

84. Wang X, Farnell YZ, Kiess AS, Peebles ED, Wamsley KGS, Zhai W. 2019. Effects of Bacillus subtilis and coccidial vaccination on caecal microbial diversity and composition of Eimeriachallenged male broilers. Poultry Science, 98(9), 3839-3849.

85. Wei Z, Zhao Y, Zhang N, Han Z, Liu X, Jiang A, Zhang Y, Wang C, Gong P, Li J, Zhang X, Yang Z. 2019. Eimeria tenella induces the release of chicken heterophil extracellular traps. Veterinary Parasitology, 275, 108931.

86. Wilkinson N, Hughes RJ, Aspden WJ, Chapman J, Moore RJ, Stanley D. 2016. The gastrointestinal tract microbiota of the Japanese quail, Coturnix japonica. Applied Microbiology and Biotechnology, 100, 4201-4209.

87. Wu Y, Zhen W, Geng Y, Wang Z, Guo Y. 2019. Effects of dietary Enterococcus faecium NCIMB 11181 supplementation on growth performance and cellular and humoral immune responses in broiler chickens. Poultry Science, 98(1), 150-163.

88. Yadav S, Jha R. 2019. Strategies to modulate the intestinal microbiota and their effects on nutrient utilization, performance, and health of poultry. Journal of Animal Science and Biotechnology, $10,2$.

89. Yan W, Sun C, Yuan J, Yang N. 2017. Gut metagenomic analysis reveals prominent roles of Lactobacillus and caecal microbiota in chicken feed efficiency. Scientific Reports, 7, 45308

90. Yang C, Kennes YM, Lepp D, Yin X, Wang Q, Yu H, Yang C, Gong J, Diarra MS. 2020. Effects of encapsulated cinnamaldehyde and citral on the performance and cecal microbiota of broilers vaccinated or not vaccinated against coccidiosis. Poultry Science, 99(2), 936-948.

91. Yang WC, Tien YJ, Chung CY, Chen YC, Chiou WH, Hsu SY, Liu HY, Liang CL, Chang CL. 2015. Effect of Bidens pilosa on infection and drug resistance of Eimeria in chickens. Research in Veterinary Science, 98, 74-81.

92. Yang WC, Yang CY, Liang YC, Yang CW, Li WQ, Chung CY, Yang MT, Kuo TF, Lin CF, Liang CL, Chang CL. 2019. Anticoccidial properties and mechanisms of an edible herb, Bidens pilosa, and its active compounds for coccidiosis. Scientific Reports., 9(1), 2896.

93. Yang WY, Lee Y, Lu H, Chou CH, Wang C. 2019. Analysis of gut microbiota and the effect of lauric acid against necrotic enteritis in Clostridium perfringens and Eimeria side-by-side challenge model. PloS One, 14(5), e0205784.

94. Zheng A, Luo J, Meng K, Li J, Bryden WL, Chang W, Zhang S, Wang LX, Liu G, Yao B. 2016. Probiotic (Enterococcus faecium) induced responses of the hepatic proteome improves metabolic efficiency of broiler chickens (Gallus gallus). BMC Genomics, 17, 1-12.

95. Zhou BH, Jia LS, Wei SS, Ding HY, Yang JY, Wang HW. 2020. Effects of Eimeria tenella infection on the barrier damage and microbiota diversity of chicken cecum. Poultry Science, 99(3), 1297-1305.

Cite this article as: Madlala T, Okpeku M \& Adeleke MA. 2021. Understanding the interactions between Eimeria infection and gut microbiota, towards the control of chicken coccidiosis: a review. Parasite 28, 48.

\section{PARASTE}

An international open-access, peer-reviewed, online journal publishing high quality papers on all aspects of human and animal parasitology

Reviews, articles and short notes may be submitted. Fields include, but are not limited to: general, medical and veterinary parasitology; morphology, including ultrastructure; parasite systematics, including entomology, acarology, helminthology and protistology, and molecular analyses; molecular biology and biochemistry; immunology of parasitic diseases; host-parasite relationships; ecology and life history of parasites; epidemiology; therapeutics; new diagnostic tools.

All papers in Parasite are published in English. Manuscripts should have a broad interest and must not have been published or submitted elsewhere. No limit is imposed on the length of manuscripts.

Parasite (open-access) continues Parasite (print and online editions, 1994-2012) and Annales de Parasitologie Humaine et Comparée (1923-1993) and is the official journal of the Société Française de Parasitologie. 